\title{
211
}

\section{IN QUEST OF THE TRANSMISSION SIZE}

\author{
LUCA G. ZAROTTI - ROBERTO PAOLUZZI \\ Institute for Earthmoving Machinery and Offroad Vehicles \\ Via Canalbianco 28, 44044 CASSANA-FERRARA (Italy)
}

\begin{abstract}
The paper addresses an automatic procedure to define the size (i.e. displacement) of the main units within a hydrostatic transmission for mobile vehicle applications. It is developed for one pump and one or more motor, and starts from a limited number of specifications: the rated engine power and speed, the overall power ratio, the number of motors, and the choice of fixed or variable displacement motor(s). Numerical results are given over a practical range of input data, and additional constraints are discussed which come from the vehicle locomotion .
\end{abstract}

\section{KEYWORDS}

Fluid Power, Transmissions, Pumps, Motors

\section{NOMENCLATURE}

$N \quad$ number of motors, -

$P \quad$ power, $\mathrm{kW}$

$p$ pressure, $\mathrm{MPa}$

$R_{t} \quad$ pump torque ratio, -

$R_{w}$ overall power ratio, -

$T$ torque, $\mathrm{Nm}$

$V \quad$ cubic displacement, $\mathrm{cm}^{3} / \mathrm{rev}$

$\alpha \quad$ displacement setting, -

$\eta \quad$ pump or motor efficiency. -

$\varphi \quad$ pump or motor speed ratio, -

$\pi \quad$ pressure ratioed to nominal, -

$\theta \quad$ speed ratioed to nominal, -

$\theta_{\alpha}$ motor overspeed function, -

$\tau$ gear(box) ratio, -

$\omega$ rotational speed, $\mathrm{rev} / \mathrm{min}$

\section{Subscripts}

$h$ hydro-mechanical (efficiency)

$m$ minimum

$n \quad$ nominal or rated



\section{INTRODUCTION}

The layout of a simple hydrostatic transmission is shown in FIGURE 1 (top), with the following parts: (1) a diesel engine; (2) a variable displacement pump with a built-in boost circuit; (3) a gearbox between engine and pump; (4) a fixed or variable displacement motor; (5) two main lines which connect pump and motor. The motor load comes from the locomotion gear of a vehicle. If two or more motors are coupled with the same pump, their output torque may be separated or merged, as shown in FIGURE 1 (bottom). 




FIGURE 1 Scheme of the hydrostatic transmission

The design of the transmission for a specific application involves two basic problems: the size of the main units, and the control law of their displacement (if applicable). The solution of the first problem is more critical because it involves the hardware cost, and constrains the whole operating envelope.

The paper introduces an automatic sizing procedure, available as starting point of more refined searches or tool for general feasibility studies. The procedure is derived in two forms: loss free and loss inclusive. In many senses, it differs from the sizing schemes offered by some fluid power manufacturers.

\section{MAIN PROCEDURE}

The aim of the sizing procedure is to find the smallest pump and motor(s) which are able to transfer the rated engine power. Then, the analysis is restricted to those operating conditions which force the engine to work at constant speed and constant torque (rated power).

\section{Mechanical Characteristic}

The loss free characteristic of a constant power transmission as mapped into the motor shaft is shown in FIGURE 2. The characteristic is bounded by two points of special interest: the maximum torque point (1) and the maximum speed point (2), which define the corner power

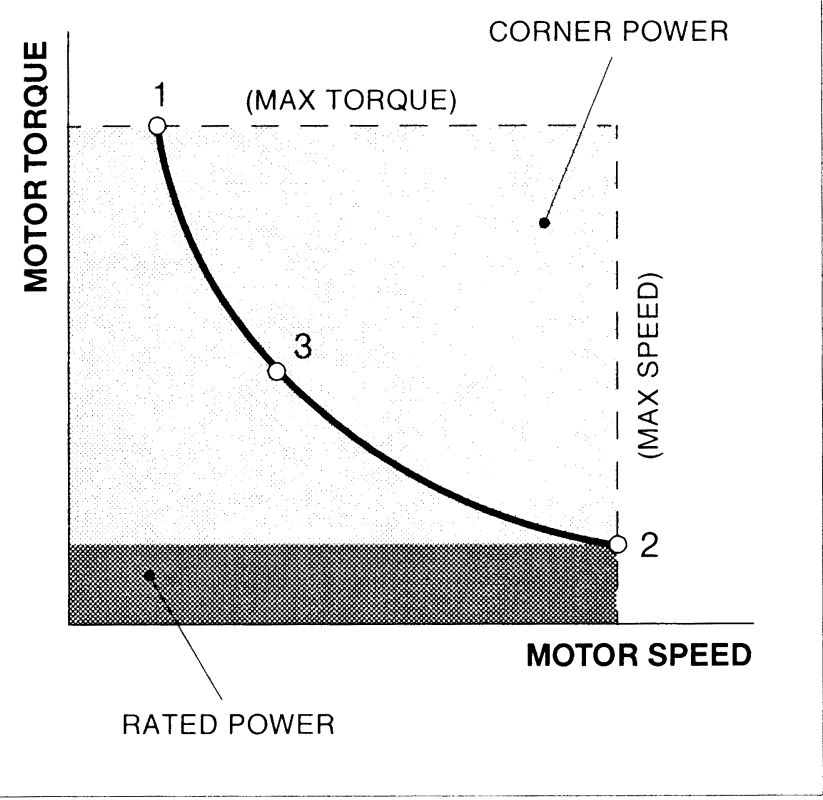

FIGURE 2 Output characteristic of the motor shaft

of the transmission. Individually, they correspond to the rated engine power, and are connected by a hyperbolic curve. In the general case of variable displacement pump and motor(s), it is useful to split the curve from point $\mathbf{1}$ to point $\mathbf{2}$ in two parts by means of an additional point $\mathbf{3}$, such that the functional parameters are the following:

- in point 1 , the pump works at minimum displacement, the motor(s) at full displacement, and the fluid is at nominal pressure;

- in point 3, the pump and the motor(s) work at full displacement, and the fluid is at minimum pressure;

- in point 2, the pump works at full displacement, the motor(s) at minimum displacement, and the fluid is at minimum pressure.

It means that the transmission is sized with a limiting control law: constant torque from point 1 to point 3 , and constant pressure from point 3 to point 2 . If the displacement of motor(s) is fixed, point $\mathbf{2}$ is coincident with point $\mathbf{3}$. In any case, point $\mathbf{2}$ and point $\mathbf{3}$ are equivalent as far as the pump is concerned.

The power ratio is usually defined as the ratio of the corner power to the rated power; in loss free conditions, it also equals the ratio of speed in point 2 to speed in point 1 , and the ratio of torque in point $\mathbf{1}$ to torque in point $\mathbf{2}$. 


\section{Procedure Inputs/Outputs}

The set of data supplied as inputs to the procedure are: the engine rated power, the engine rated speed, the overall power ratio, and the number of motors. The main outputs are: the pump displacement, the motor(s) displacement, and the gear(box) ratio.

The power ratio is used because strictly related with the vehicle locomotion, and in some way representative of the vehicle class. For instance, in [1] three ranges of this parameter are evidenced: from 3 to 5 (light special duty vehicles), from 5 to 8 (light vanes, tractors, light passenger vehicles), and from 8 to 12 (heavy lorries, buses, earthmoving equipment).

\section{Pump and Motor Efficiency}

Volumetric and hydro-mechanical losses in the main units are taken into account through the relevant efficiencies. It is assumed here that they are described by a set of functional models with the generic form $\eta(\pi, \theta, \alpha)$. Models of this type are found, for instance, in [2]. No explicit dependance on the unit size is considered.

\section{FIRST STAGE (PUMP SIDE)}

The first stage of the procedure involves two parameters: the gear ratio and the torque ratio. Stated that the engine works at its rated speed, and the pump has the minimum size if working at its nominal speed, the gear ratio is

$$
\tau=\frac{\omega_{n}^{E}}{\omega_{n}^{P}}
$$

The torque ratio involves the (full) displacement of the actual pump and the displacement of the matched pump, i.e. the particular pump whose nominal torque equals the rated engine torque

$$
R_{t}=\frac{V^{P}}{V_{z}^{P}}
$$

The name "torque ratio" is used because EQ. 2 equals the ratio of the pump nominal torque to the engine rated torque.

\section{Primary Outputs}

The published data of commercial units suggest that the nominal speed of the axial piston pumps decrease exponentially with size, i.e.

$$
\omega \cdot V^{\varepsilon}=\text { const }
$$

Based on that, a relationship is derived, which provides a link (independent of efficiency) between EQ. 1 and EQ. 2

$$
\tau=\frac{1}{\varphi^{P}} \cdot R_{t}^{\varepsilon}
$$

and includes the pump speed ratio between the nominal speed of the matched pump, and the rated engine speed. A useful value of $\varepsilon$ has been found to be " $\log 2 "$ (the nominal speed halves when the displacement is ten times larger).

The pump speed ratio is computed independently by introducing a reference pump (whose data are completely known), which is representative of the current technology

$$
\varphi^{P}=\left(\frac{P_{r}}{P_{n}^{E}}\right)^{\varepsilon} \cdot\left(\frac{\omega_{r}}{\omega_{n}^{E}}\right)^{1-\varepsilon}
$$

(the relevant proof is given in [3]). Based on the same reasoning, the pump displacement is computed as

$$
V^{P}=V_{r} \cdot \tau^{1 / \varepsilon} \cdot\left(\frac{\omega_{r}}{\omega_{n}^{E}}\right)^{1 / \varepsilon}
$$

Once the torque ratio and the engine data are known, EQ. 6, with EQ. 5 and EQ. 4, are sufficient to solve the first stage of the sizing problem.

\section{Secondary Outputs}

Efficiencies play an explicit role if two secondary parameters are derived, i.e. the minimum pressure (occurring in point $\mathbf{2}$ and $\mathbf{3}$ of FIGURE 2) and the minimum pump displacement (occurring in point $\mathbf{1}$ ). The relevant equations are

$$
\begin{aligned}
& \pi_{m}=\frac{\tau}{R_{t}} \cdot \frac{\eta_{h}^{P}\left(\pi_{m}, 1,1\right)}{\eta_{h}^{P}(1,1,1)}=\frac{\tau}{R_{t}} \cdot \frac{\eta_{2 h}^{P}}{\eta_{0 h}^{P}} \\
& \alpha_{m}^{P}=\frac{\tau}{R_{t}} \cdot \frac{\eta_{h}^{P}\left(1,1, \alpha_{m}^{P}\right)}{\eta_{h}^{P}(1,1,1)}=\frac{\tau}{R_{t}} \cdot \frac{\eta_{1 h}^{P}}{\eta_{0 h}^{P}}
\end{aligned}
$$

Both equations are implicit; otherwise, if efficiency is disregarded, the minimum pressure and the minimum pump displacement are the same.

\section{SECOND STAGE (MOTOR SIDE)}

The second stage of the procedure deals with the size of the hydraulic motors, and is based on the 
continuity equation between pump and motors in point 2 (FIGURE 2).

\section{Basic Outputs}

Stated that the motor size is the smallest if its nominal speed is reached in point $\mathbf{2}$, the loss free continuity equation is

$$
N \cdot V^{M} \cdot \omega_{n}^{M} \cdot \alpha_{m}^{M} \cdot \theta_{\alpha}=V^{P} \cdot \omega_{n}^{P}
$$

By introducing the volumetric ratio of the individual motor displacement to the pump displacement

$$
R_{v}=\frac{V^{M}}{V^{P}}
$$

and expressing the nominal speed of pump and motors through the relevant speed ratios (the motor speed ratio is defined in the same way as the pump speed ratio)

$$
\frac{\omega_{n}^{P}}{\omega_{n}^{E}}=\varphi^{P} \cdot\left(\frac{V_{z}^{P}}{V^{P}}\right)^{\varepsilon} \quad \frac{\omega_{n}^{M}}{\omega_{n}^{E}}=\varphi^{M} \cdot\left(\frac{V_{z}^{P}}{V^{M}}\right)^{\varepsilon}
$$

after some transformations, the following result is finally available

$$
R_{v}^{1-\varepsilon}=\frac{\varphi^{P}}{\varphi^{M}} \cdot \frac{1}{\alpha_{m}^{M} \cdot \theta_{\alpha} \cdot N}
$$

Once the number of motors and the minimum motor displacement are known, EQ. 11 solves the second stage of the sizing problem: in fact, the motor size is found explicitly as

$$
V^{M}=R_{v} \cdot V^{P}
$$

If efficiencies are taken into account, the continuity equation changes. Then, EQ. 11 is no longer true, and should be modified as follows

$$
R_{v}^{1-\varepsilon}=\frac{\varphi^{P}}{\varphi^{M}} \cdot \frac{1}{\alpha_{m}^{M} \cdot \theta_{\alpha} \cdot N} \cdot \frac{\eta_{v}^{P}\left(\pi_{m}, 1,1\right)}{\eta_{v}^{M}\left(\pi_{m}, \theta_{\alpha}, \alpha_{m}^{M}\right)}
$$

which is implicit because requires the minimum pressure from EQ. 7. A secondary parameter is also affected by efficiencies, i.e. the minimum motor speed, which comes from the continuity equation in point 1 (FIGURE 2)

$$
\theta_{m}^{M}=\alpha_{m}^{M} \cdot \theta_{\alpha} \cdot \alpha_{m}^{P} \cdot \frac{\eta_{2 v}^{M}}{\eta_{2 v}^{P}} \cdot \frac{\eta_{1 v}^{P}}{\eta_{1 v}^{M}}
$$

The solution is implicit because the efficiencies on the right side depend on $\theta_{m}^{M}, \alpha_{m}^{M}, \theta_{\alpha}, \alpha_{m}^{P}, \pi_{m}$.

\section{Complementary Remarks}

The motor speed ratio is not computed explicitly through a reference motor, but is derived from the pump speed ratio as

$$
\varphi^{M}=\kappa \cdot \varphi^{P} \quad \kappa>1
$$

where $\kappa$ takes into account that the nominal speed of motors is generally higher than the nominal speed of pumps of the same size.

The motor overspeed function $\theta_{\alpha}$ takes into account that the nominal speed of variable motors increases at partial displacement settings. Theoretically, the function equals the inverse of the minimum displacement but is affected by an upper bound (30\% overspeed is chosen in this case), i.e.

$$
\theta_{\alpha}=\frac{1}{\alpha_{m}^{M}} \quad 1 \leq \theta_{\alpha} \leq 1.3
$$

If better data are available, both functional dependance and boundary may be changed.

\section{THIRD STAGE (COORDINATION)}

The first and second stage of the procedure need the specification of two parameters: the torque ratio, and the minimum motor displacement (if variable). The missing link is provided by the overall power ratio, though the effect of losses is to be considered separately.

\section{First Case (Loss Free)}

The definiton of the power ratio leads to the following relationship (which does not depend on the number of motors)

$$
R_{w}=\frac{R_{t}}{\alpha_{m}^{M} \cdot \tau}=\frac{1}{\alpha_{m}^{M}} \cdot \frac{1}{\alpha_{m}^{P}}
$$

In the simple case of constant displacement motor(s), the solution is straightforward: in fact, EQ. 17 becomes

$$
R_{t}=\tau \cdot R_{w}
$$

and EQ. 4 allows the explicit solution for the gear(box) ratio.

Conversely, in the general case of variable displacement motor(s), a decision has to be taken, considering that EQ. 17 depends on the extension of the displacement control in pump and motor(s). Among the infinite number of options, it seems reasonable to assume that the minimum displacement of pump and motor(s) are the 
same, which gives the following results

$$
\alpha_{m}^{M}=\frac{1}{\sqrt{R_{w}}} \quad R_{t}=\tau \cdot \sqrt{R_{w}}=\left(\frac{\sqrt{R_{w}}}{\varphi^{P}}\right)^{\frac{1}{1-\varepsilon}}
$$

to be used in the first and second stage of the procedure. Practically, the minimum motor displacement is not free, and the procedure uses the following range

$$
0.3 \leq \alpha_{m}^{M}<1
$$

If EQ. 19 crosses the lower boundary of EQ. 20, the solution is saturated, and EQ. 19 is to be modified accordingly.

\section{Second Case (With Losses)}

If efficiencies are considered, the definitions of the overall power ratio in terms of power, torque or speed are not equivalent.

If the first option is adopted (ratio of corner and rated power), EQ. 17 becomes

$$
R_{w}=\frac{1}{\alpha_{m}^{M}} \cdot \frac{1}{\alpha_{m}^{P}} \cdot \frac{\eta_{2 v}^{P}}{\eta_{2 v}^{M}} \cdot \eta_{1 h}^{M} \cdot \eta_{1 h}^{P}
$$

If the second option is adopted, i.e. ratio of torque in point $\mathbf{1}$ and point $\mathbf{2}$ (FIGURE 2), EQ. 17 becomes

$$
R_{w}=\frac{1}{\alpha_{m}^{M}} \cdot \frac{1}{\alpha_{m}^{P}} \cdot \frac{\eta_{1 h}^{P}}{\eta_{2 h}^{P}} \cdot \frac{\eta_{1 h}^{M}}{\eta_{2 h}^{M}}
$$

The efficiency terms on the right side of EQ. 21 and EQ. 22 depend on $\theta_{m}^{M}, \alpha_{m}^{M}, \theta_{\alpha}, \alpha_{m}^{P}, \pi_{m}$.

\section{FIXED OR VARIABLE DISPLACEMENT}

The procedure requires the preliminary choice of variable or fixed displacement motors. The existence of a unique criterion is an open problem, still waiting for a general solution. Clearly, it should depend on the power ratio (fixed displacement at low power ratios, and variable displacement at high power ratios), but it is still discussed empirically: for instance, in [1] and [4] the shift point is located at $R_{w}=3.0$ and $R_{w}=2.5$ respectively, without further explanations. A better approach would be probably based either on efficiency (which decrease as the displacement setting decreases) or pump and motor cost (which increase with size, and are higher for variable displacement units). Provisional results from simple cost models confirm that it is reasonable to accept a shift point located somewhere between 2 and 3 .

\section{EXAMPLE}

As an example of the procedure, the size of units within a transmission with one pump and one motor are plotted in FIGURE 3 and FIGURE 4. The nominal pressure is $40 \mathrm{Mpa}$, and the reference pump has the following data (to be used in EQ. 5 and EQ. 6)

$$
\omega_{r}=2500 \quad V_{r}=130 \quad P_{r}=216.7
$$

Two values of the rated engine power are considered (50 and $100 \mathrm{~kW} @ 2500 \mathrm{rpm}$ ), and the power ratio ranges from 1 to 10 . The solid lines refer to a fixed displacement motor, whereas the

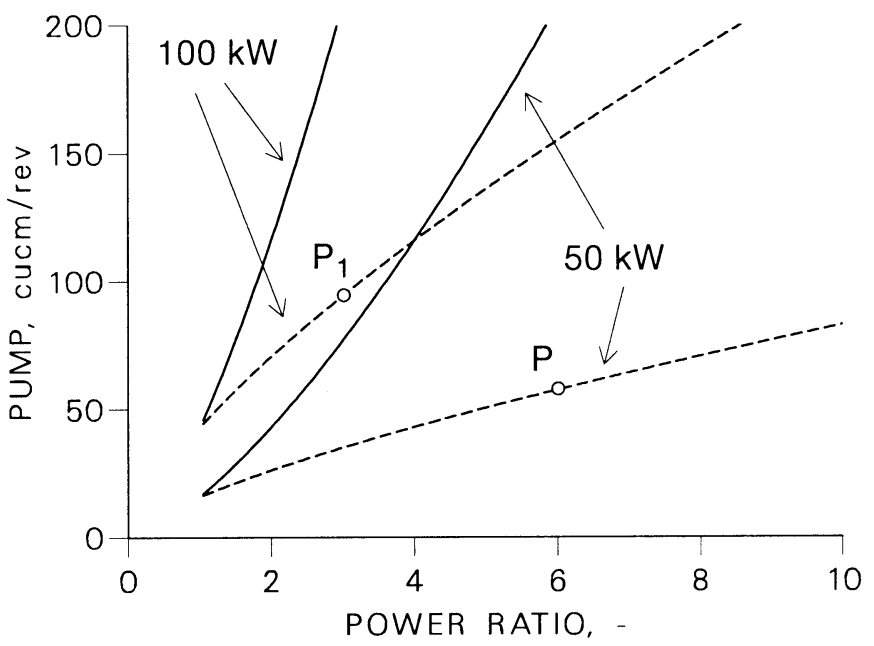

FIGURE 3 Pump displacement vs power ratio

dashed lines refer to a variable displacement motor. Right of points labelled as X (FIGURE 4) the overspeed function is bounded. It is ramarkable that the pump size (FIGURE 3 ) is independent of the number of motors.

The size of pump and motor which correspond to the fixed displacement option are not much different each other and increase considerably with the power ratio (at $100 \mathrm{~kW}$, the displacement of $200 \mathrm{~cm}^{3} / \mathrm{rev}$ is reached around 3 for both). Conversely, the variable displacement option allows for much smaller pumps (at 100 $\mathrm{kW}$, the displacement of $200 \mathrm{~cm}^{3} / \mathrm{rev}$ is reached between 8 and 9), but little smaller motors (at $100 \mathrm{~kW}$, the displacement of $200 \mathrm{~cm}^{3} / \mathrm{rev}$ is reached around 4). This seems a good reason to use two motors instead of one: in fact, the size 


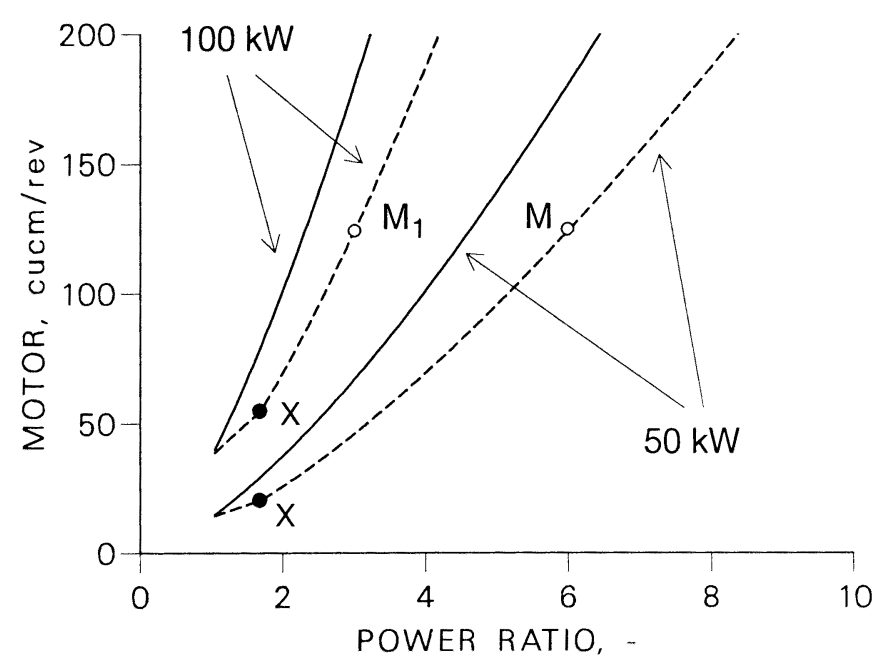

FIGURE 4 Motor displacement vs power ratio

of multiple motors decrease more than proportionally

$$
V^{M} \propto \frac{1}{N^{\frac{1}{1-\varepsilon}}} \quad \frac{1}{1-\varepsilon}>1
$$

all other conditions being the same. In practice, with two motors the threshold of $200 \mathrm{~cm}^{3} / \mathrm{rev}$ is crossed at 5 instead of 4 .

\section{VEHICLE CONSTRAINTS}

The input data of the procedure are influenced by the constraints due to the application environment, i.e. the vehicle locomotion.

Firstly, the maximum torque (point $\mathbf{1}$ in FIGURE 2 ) is limited by the traction capability. If the vehicle reaches its maximum speed $v_{\max }$ in point 2 (FIGURE 2), the power ratio is approximately limited (for a loss free transmission) by the inequality

$$
R_{w} \leq \mu_{t} \cdot v_{\max } \cdot \lambda \cdot \frac{W}{P_{n}^{E}}
$$

being $W$ the total vehicle weight, $\lambda$ the weight portion supported by the driving axle, and $\mu_{t}$ the traction coefficient. For example, if the engine power is $50 \mathrm{~kW}$, and the limiting power ratio is 6 , the pump and motor displacement are given by point $\mathbf{P}$ and $\mathbf{M}$ in FIGURE 3 and FIGURE 4 (the motor displacement is variable because the power ratio is above 3 ). If the engine power becomes $100 \mathrm{~kW}$, the limiting power ratio for the same vehicle becomes 3 , and the pump and motor displacement are given by point $\mathbf{P}_{\mathbf{1}}$ and $\mathbf{M}_{\mathbf{1}}$ in FIGURE 3 and FIGURE 4 (a larger pump and the same motor).

Secondly, the torque at maximum speed must be greater than the motion resistance on flat and level ground. This condition limits the specific power of the vehicle

$$
\frac{W}{P_{n}^{E}} \leq \frac{1}{\mu_{r} \cdot v_{\max }}
$$

being $\mu_{r}$ the resistance coefficient. If EQ. 25 and EQ. 26 are combined, a new expression of the limiting power ratio is derived

$$
R_{w} \leq \frac{\mu_{t}}{\mu_{r}} \cdot \lambda
$$

which is independent of the vehicle speed, but seems more useful in theory than in practice.

\section{CONCLUSIONS}

The feasibility has been proved of an automatic procedure, which is able to find in closed or iterative form the minimum size of pump and motor (fixed or variable displacement) in a hydrostatic transmission for mobile applications, once a limited number of inputs are available in connection with the relevant locomotion constraints.

Further investigations are to be carried out in order to identify reliable criteria to assist the option for fixed or variable displacement motor(s).

\section{REFERENCES}

1. C.M.Edghill, Some Factors Determining the Choice of a Particular Hydrostatic Transmission Unit, Proc. of the 1st Fluid Power Symposium, BHRA, Cranfield, 1969, pp. 73-106

2. G.L.Zarotti, and N.Nervegna, Pump Efficiencies Approximation and Modelling, Proc. of the 6th International Fluid Power Symposium, BHRA Fluid Engineering, Cambridge, 1981, pp. 145-164

3. R. Paoluzzi, and G.L.Zarotti, Hydrostatic Transmissions: a Procedure for Component Parameter Definition, CEMOTER-CNR Internal Report, 1991, Nr.349

4. F.W.Pollman, Application of Hydrostatic Transmissions to Increase User Value, SAE paper 841101, Society of Automotive Engineers, 1984 